\title{
PHYTOSEIIDAE (ACARI: MESOSTIGMATA) ON PLANTS OF THE CENTRAL REGION OF THE BRAZILIAN CERRADO
}

\author{
José Marcos ReZende ${ }^{1 *}$ and Antonio Carlos LOFEGO ${ }^{2}$ \\ (Received 08 July 2011; accepted 30 September 2011; published online 20 December 2011) \\ ${ }^{1}$ PPG - Biologia Animal, UNESP-Universidade Estadual Paulista, São José do Rio Preto, SP, Brazil; \\ jmrezende@live.com ( ${ }^{*}$ Corresponding author) \\ ${ }^{2}$ Depto. de Zoologia e Botânica, UNESP-Universidade Estadual Paulista, Rua Cristóvão Colombo, 2265, Jardim Nazareth, \\ 15054-000, São José do Rio Preto, SP, Brazil; aclofego@ig.com.br
}

\begin{abstract}
Four surveys of Phytoseiidae species associated with native plants were conducted in ten fragments of Cerrado sensu stricto to determine the species associated with the plants of this region. Twelve of the most common plant species were sampled in each fragment. Twenty six phytoseiid species were recorded during the surveys. Stryphnodendron adstringens was the host plant that had the highest number of species (eight species). Amblyseius neochiapensis Lofego, Moraes and McMurtry, 2000 and Neoseiulus tunus (De Leon), 1967 were the most common phytoseiid species. These species occurred on 24 and 20 host species, respectively. Approximately $76 \%$ of the phytoseiids found belonged to the subfamily Amblyseiinae Muma, 1961. Almost $27 \%$ of phytoseiid species recorded were associated with only one plant species. In addition, approximately two thirds of species found in this study also occur in the Atlantic Forest. The comparison of our results from the central areas with previous surveys in peripheral areas of the Cerrado showed that only 12 phytoseiid species were common to both areas.
\end{abstract}

KEYWORDS - survey; mites; Neotropic region; Phytoseiidae; Brazil

\section{INTRODUCTION}

Many species of Phytoseiidae Berlese, 1913 are predators, some of which proved to be highly effective in controlling pest mites in various crops (McMurtry and Croft 1997; Moraes and Flechtmann 2008). Therefore, there is a strong need for more and comprehensive studies about phytoseiid species.

Surveys on Phytoseiidae in specific regions, biomes or crops constitute the first step to further studies about the use of predatory mites in the biological control (Gerson et al. 2003). Many surveys like these have been conducted in South America, being the bases for important programs to control of pest mites such as Aceria guerreronis Keifer, 1965 (Gondim Jr. and Moraes 2001; Lawson-Balagbo et al. 2008), Mononychellus tanajoa (Bondar, 1938) (Farias et al. 1981; Moraes and McMurtry 1983; Moraes et al. 1991) and Tetranychus evansi Baker and Pritchard, 1960 (Furtado et al. 2004[2005]; 2006; 2007; Fiaboe et al. 2007; Guanilo et al. 2008c; 2010; Rosa et al. 2005).

In Brazil, surveys on phytoseiid species from natural areas are mostly concentrated in areas of Atlantic Forest of state of São Paulo (Buosi et al. 2006; Castro and Moraes 2010; Daud et al. 2007; Demite et al. 2011; Feres and Moraes 1998; Feres and Nunes 2001; Feres et al. 2005; Zacarias and Moraes 2002). 


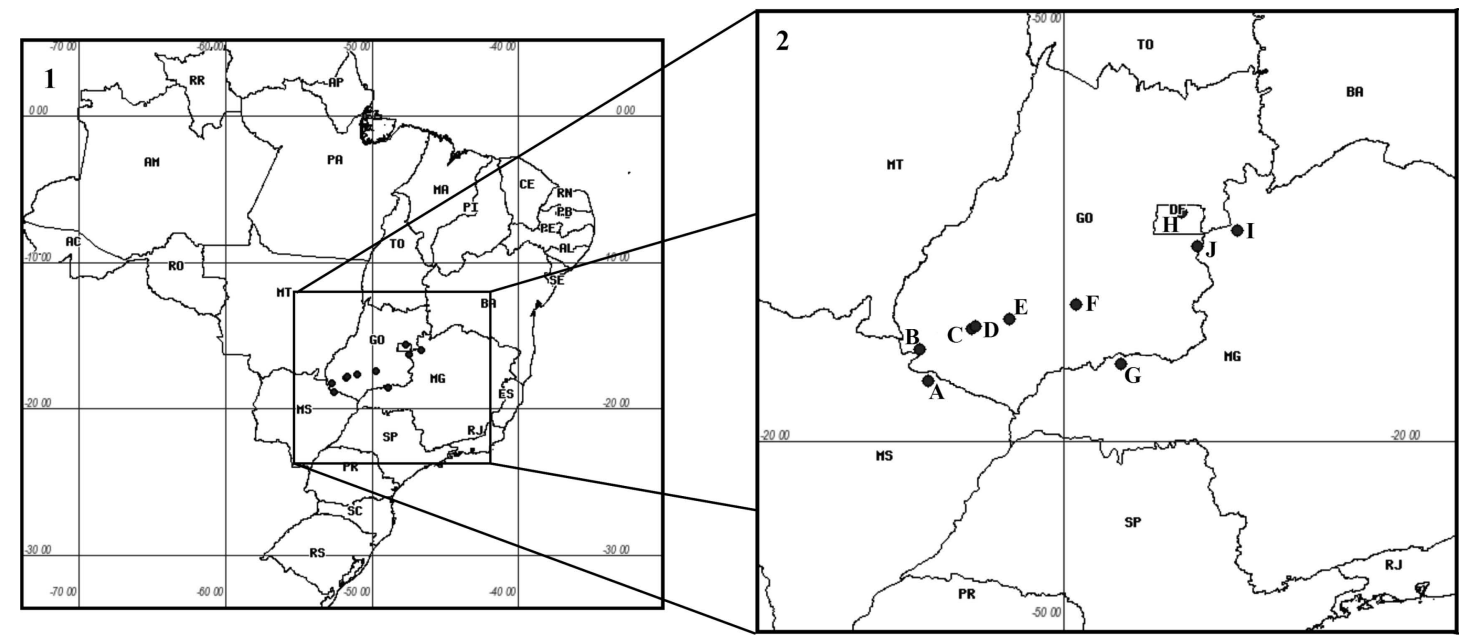

FIGURE 1: Location of study areas. 1 - Map of Brazil highlighting the region sampled. 2 - detailed map, with location of study points. Letters correspond to cities in the sampling areas: (A: Chapadão do Sul-MS, B: Chapadão do Céu-GO, C: Jataí-GO, D: Jataí-GO, E: Rio Verde-GO, F: Edealina-GO, G: Tupaciguara-MG, H: Brasília-DF, I: Unai, MG and J: Cristalina-GO).

Though the Cerrado is the second largest Brazilian biome, surveys were made only on peripheral areas (Lofego et al. 2004; Lofego and Moraes 2006; Demite et al. 2009) and so far no information is available on these predators in its central areas.

Moreover, the Cerrado is currently threatened by the rapid increase in agricultural activities and constant loss of natural areas (Ministério do Meio Ambiente 2007), which may consequently threat its characteristic mite fauna. So, the objective of this study was to do the inventory of phytoseiid species associated with native plants of this biome, specifically in fragments of cerrado sensu stricto.

\section{MATERIALS AND METHODS}

\section{Study areas}

This study was conducted in ten fragments of Cerrado, located in southeastern and central Brazil (Figure 1, Table 1). The climate in the region is characterized as Aw of Köppen (Ribeiro and Walter 1998) with two distinct seasons: a rainy season (October to March) and a dry season (April to September). The chosen areas are situated in the core region of biome, considered the original distribution of the Cerrado (Ministério do Meio Ambiente 2007).

\section{Sampling}

Samples were taken in fragments between October 2009 and June 2010. Twelve species of more abundant native plants were sampled in each area, belonging to different strata and plant families. Abundance of plants was evaluated visually. At least two individuals of each plant species selected were sampled, in total 57 plant species, in all fragments (Table 1).

The material collected from each plant species consist of leaves and flowers (when present). We used the following procedure for mite extraction: firstly, the material was washed separately in buckets containing eight liters of $30 \%$ ethanol. After each wash, the ethanol was filtered through sieves with $25 \mu \mathrm{m}$ nylon mesh. With a wash-bottle, the debris on the mesh was washed with $67 \%$ ethanol into vials filled with the same alcohol for preservation. These procedures were done in the fragments, immediately after the samplings. So, the vials were placed in plastic boxes and brought to laboratory by car.

The material was screened under a stereoscopic microscope in laboratory. Phytoseiids found were mounted in Hoyer's medium (Moraes and Flechtmann 2008) and the slides labelled. The collection data is indicated in the following order for 
TABLE 1: Areas of study with the plant species sampled for the survey of Phytoseiidae. Brazilian Cerrado 2009/2010.

\begin{tabular}{|c|c|c|c|}
\hline Area code & Location & Coordenates & Sampled plants \\
\hline A & Chapadão do Sul-MS & $52^{\circ} 35^{\prime} \mathrm{W} 18^{\circ} 51^{\prime} \mathrm{S}$ & $\begin{array}{l}\text { Bauhinia sp. } 1 \text { (Fabaceae) } \\
\text { Byrsonima intermedia A. Juss. (Malphigiaceae) } \\
\text { Byrsonima pachyphylla A. Juss. (Malphigiaceae) } \\
\text { Campomanesia pubescens (DC.) Berg (Myrtaceae) } \\
\text { Caryocar brasiliense Camb. (Caryocaraceae) } \\
\text { Didymopanax vinosum Cham. \& Schltdl. (Araliaceae) } \\
\text { Fuirena umbellata Rottb. (Cyperaceae) } \\
\text { Miconia albicans (Sw.) Triana (Melastomataceae) } \\
\text { Myrtaceae sp. } 1 \text { (Myrtaceae) } \\
\text { Myrtaceae sp. } 2 \text { (Myrtaceae) } \\
\text { Pouteria torta (Mart.) Radlk. (Sapotaceae) } \\
\text { Roupala brasiliensis Klotzsch (Proteaceae) }\end{array}$ \\
\hline B & Chapadão do Céu-GO & $52^{\circ} 44^{\prime} \mathrm{W} 18^{\circ} 15^{\prime} \mathrm{S}$ & $\begin{array}{l}\text { Bauhinia sp. } 2 \text { (Fabaceae) } \\
\text { Campomanesia pubescens (DC.) Berg (Myrtaceae) } \\
\text { Caryocar brasiliense Camb. (Caryocaraceae) } \\
\text { Didymopanax vinosum Cham. \& Schltdl. (Araliaceae) } \\
\text { Fuirena umbellata Rottb. (Cyperaceae) } \\
\text { Harconia speciosa Gom. (Apocynaceae) } \\
\text { Miconia albicans (Sw.) Triana (Melastomataceae) } \\
\text { Myrtaceae sp. } 1 \text { (Myrtaceae) } \\
\text { Myrtaceae sp. } 2 \text { (Myrtaceae) } \\
\text { Myrtaceae sp. } 3 \text { (Myrtaceae) } \\
\text { Qualea grandiflora Mart. (Vochysiaceae) } \\
\text { Xylopia aromatica (Lam.) Mart. (Annonaceae) }\end{array}$ \\
\hline $\mathrm{C}$ & Jataí-GO & $51^{\circ} 45^{\prime} \mathrm{W} 17^{\circ} 51^{\prime} \mathrm{S}$ & $\begin{array}{l}\text { Byrsonima pachyphylla A. Juss. (Malphigiaceae) } \\
\text { Caryocar brasiliense Camb. (Caryocaraceae) } \\
\text { Cf. Dilleniaceae sp. (Dilleniaceae) } \\
\text { Dimorphandra mollis Benth. (Fabaceae) } \\
\text { Genipa americana L. (Rubiaceae) } \\
\text { Melinis minutiflora P. Beauv. (Poaceae) } \\
\text { Miconia albicans (Sw.) Triana (Melastomataceae) } \\
\text { Qualea grandiflora Mart. (Vochysiaceae) } \\
\text { Stryphnodendron adstringens (Mart.) Coville (Fabaceae) } \\
\text { Tocoyena formosa (Cham. \& Schltdl.) K.Schum. (Rubiaceae) } \\
\text { Trachipogon sp. (Poaceae) } \\
\text { Xylopia aromatica (Lam.) Mart. (Annonaceae) }\end{array}$ \\
\hline
\end{tabular}


TABLE 1: Continued.

\begin{tabular}{|c|c|c|c|}
\hline Area code & Location & Coordenates & Sampled plants \\
\hline $\mathrm{D}$ & Jataí-GO & $51^{\circ} 41^{\prime} \mathrm{W} 17^{\circ} 49^{\prime} \mathrm{S}$ & $\begin{array}{l}\text { Anacardium occidentale L. (Anacardiaceae) } \\
\text { Byrsonima coccolobifolia Kunth (Malphigiaceae) } \\
\text { Byrsonima pachyphylla A. Juss. (Malphigiaceae) } \\
\text { Caryocar brasiliense Camb. (Caryocaraceae) } \\
\text { Cf. Dilleniaceae sp. (Dilleniaceae) } \\
\text { Dimorphandra mollis Benth. (Fabaceae) } \\
\text { Harconia speciosa Gom. (Apocynaceae) } \\
\text { Miconia albicans (Sw.) Triana (Melastomataceae) } \\
\text { Plenckia populnea Reissek (Celastraceae) } \\
\text { Qualea grandiflora Mart. (Vochysiaceae) } \\
\text { Tabebuia aurea (J.Silva Manso) Benth. \& Hook. (Bignoniaceae) } \\
\text { Trachipogon sp. (Poaceae) }\end{array}$ \\
\hline $\bar{E}$ & Rio Verde-GO & $51^{\circ} 02^{\prime} \mathrm{W} 17^{\circ} 40^{\prime} \mathrm{S}$ & $\begin{array}{l}\text { Xylopia aromatica (Lam.) Mart. (Annonaceae) } \\
\text { Byrsonima coccolobifolia Kunth (Malphigiaceae) } \\
\text { Byrsonima intermedia A. Juss. (Malphigiaceae) } \\
\text { Caryocar brasiliense Camb. (Caryocaraceae) } \\
\text { Davilla elliptica A. St.-Hil. (Dilleniaceae) } \\
\text { Doliocarpus cf. dentatus Standl. (Dilleniaceae) } \\
\text { Genipa americana L. (Rubiaceae) } \\
\text { Himatanthus obovatus (Müll.Arg.) Woodson (Apocynaecae) } \\
\text { Matayba elaeagnoides Radlk. (Sapindaceae) } \\
\text { Miconia albicans (Sw.) Triana (Melastomataceae) } \\
\text { Ouratea spectabilis (Mart.) Engl. (Ochnaceae) } \\
\text { Sclerobium paniculatum Vogel (Fabaceae) }\end{array}$ \\
\hline $\bar{F}$ & Edealina-GO & $49^{\circ} 45^{\prime} \mathrm{W} 17^{\circ} 24^{\prime} \mathrm{S}$ & $\begin{array}{l}\text { Alibertia edulis (A. Rich.) A. Rich. (Rubiaceae) } \\
\text { Astronium sp. (Anacardiaceae) } \\
\text { Bauhinia sp. } 3 \text { (Fabaceae) } \\
\text { Byrsonima verbascifolia (L.) Rich. ex A.Juss. (Malphigiaceae) } \\
\text { Caryocar brasiliense Camb. (Caryocaraceae) } \\
\text { Davilla elliptica A. St.-Hil. (Dilleniaceae) } \\
\text { Erythroxylum deciduum A. St.-Hil. (Erythroxylaceae) } \\
\text { Guettarda viburnoides Cham. \& Schltdl. (Rubiaceae) } \\
\text { Qualea grandiflora Mart. (Vochysiaceae) } \\
\text { Rhamnidium elaeocarpum Reissek (Rhamnaceae) } \\
\text { Roupala brasiliensis Klotzsch (Proteaceae) } \\
\text { Solanum lycocarpum St. Hil. (Solanaceae) }\end{array}$ \\
\hline
\end{tabular}


TABLE 1: Continued.

\begin{tabular}{|c|c|c|c|}
\hline Area code & Location & Coordenates & $\begin{array}{c}\text { Sampled plants } \\
\end{array}$ \\
\hline$G$ & Tupaciguara-MG & $48^{\circ} 54^{\prime} \mathrm{W} 18^{\circ} 31^{\prime} \mathrm{S}$ & $\begin{array}{l}\text { Caryocar brasiliense Camb. (Caryocaraceae) } \\
\text { Cf. Dilleniaceae sp. (Dilleniaceae) } \\
\text { Cf. Moraceae sp. (Moraceae) } \\
\text { Davilla elliptica A. St.-Hil. (Dilleniaceae) } \\
\text { Miconia albicans (Sw.) Triana (Melastomataceae) } \\
\text { Qualea grandiflora Mart. (Vochysiaceae) } \\
\text { Roupala brasiliensis Klotzsch (Proteaceae) } \\
\text { Sclerobium paniculatum Vogel (Fabaceae) } \\
\text { Stryphnodendron adstringens (Mart.) Coville (Fabaceae) } \\
\text { Tabebuia ochracea (Cham.) Standley (Bignoniaceae) } \\
\text { Vochysia cinnamomea Pohl (Vochysiaceae) } \\
\text { Xylopia aromatica (Lam.) Mart. (Annonaceae) }\end{array}$ \\
\hline $\mathrm{H}$ & Brasília-DF & $47^{\circ} 44^{\prime} \mathrm{W} 15^{\circ} 38^{\prime} \mathrm{S}$ & $\begin{array}{l}\text { Brosimum gaudichaudii Trécul. (Moraceae) } \\
\text { Caryocar brasiliense Camb. (Caryocaraceae) } \\
\text { Cf. Dilleniaceae sp. (Dilleniaceae) } \\
\text { Echinolaena inflexa (Poir.) Chase (Poaceae) } \\
\text { Hymenaea stigonocarpa Mart. ex Hayne (Fabaceae) } \\
\text { Kielmeyera sp. (Clusiaceae) } \\
\text { Palicourea rigida Kunth (Rubiaceae) } \\
\text { Plenckia populnea Reissek (Celastraceae) } \\
\text { Qualea grandiflora Mart. (Vochysiaceae) } \\
\text { Rapanea sp. (Myrsinaceae) } \\
\text { Roupala brasiliensis Klotzsch (Proteaceae) } \\
\text { Stryphnodendron adstringens (Mart.) Coville (Fabaceae) } \\
\text { Trachipogon sp. (Poaceae) }\end{array}$ \\
\hline $\bar{I}$ & Unaí-MG & $46^{\circ} 41^{\prime} \mathrm{W} 15^{\circ} 59^{\prime} \mathrm{S}$ & $\begin{array}{l}\text { Byrsonima coccolobifolia Kunth (Malphigiaceae) } \\
\text { Caryocar brasiliense Camb. (Caryocaraceae) } \\
\text { Cf. Dilleniaceae sp. (Dilleniaceae) } \\
\text { Chamaecrista orbiculata (Benth.) H. S. Irwin \& Barneby (Fabaceae) } \\
\text { Duguetia furfuracea (A. St.-Hil.) Benth. \& Hook.f. (Annonaceae) } \\
\text { Echinolaena inflexa (Poir.) Chase (Poaceae) } \\
\text { Kielmeyera cf. coriacea (Spreng.) Mart. (Clusiaceae) } \\
\text { Ouratea spectabilis (Mart.) Engl. (Ochnaceae) } \\
\text { Pouteria ramiflora (Mart.) Radlk. (Sapotaceae) } \\
\text { Qualea grandiflora Mart. (Vochysiaceae) } \\
\text { Rapanea sp. (Myrsinaceae) } \\
\text { Stryphnodendron adstringens (Mart.) Coville (Fabaceae) }\end{array}$ \\
\hline
\end{tabular}


TABLE 1: Continued.

\begin{tabular}{|c|c|c|c|}
\hline Area code & Location & Coordenates & Sampled plants \\
\hline $\mathrm{J}$ & Cristalina-GO & $47^{\circ} 27^{\prime} \mathrm{W} 16^{\circ} 17^{\prime} \mathrm{S}$ & $\begin{array}{l}\text { Annona crassiflora Mart. (Annonaceae) } \\
\text { Byrsonima coccolobifolia Kunth (Malphigiaceae) } \\
\text { Caryocar brasiliense Camb. (Caryocaraceae) } \\
\text { Cf. Dilleniaceae sp. (Dilleniaceae) } \\
\text { Davilla elliptica A. St.-Hil. (Dilleniaceae) } \\
\text { Kielmeyera cf. coriacea (Spreng.) Mart. (Clusiaceae) } \\
\text { Ouratea spectabilis (Mart.) Engl. (Ochnaceae) } \\
\text { Piptocarpha rotundifolia (Less.) Baker (Asteraceae) } \\
\text { Qualea grandiflora Mart. (Vochysiaceae) } \\
\text { Stryphnodendron adstringens (Mart.) Coville (Fabaceae) } \\
\text { Tabebuia ochracea (Cham.) Standley (Bignoniaceae) } \\
\text { Trachipogon sp. (Poaceae) }\end{array}$ \\
\hline
\end{tabular}

each species: locality (in bold), host name, date of sample, and number of individuals in parentheses. The mites are deposited in the Acari collection (DZSJRP), Department of Zoology and Botany, Universidade Estadual Paulista (UNESP), São José do Rio Preto, São Paulo, available at: http://www.splink.cria.org.br.

\section{RESULTS}

Twenty six phytoseiid species belonging to thirteen genera were recorded. Amblyseius neochiapensis Lofego, Moraes and McMurtry, 2000 was the most common species, occurring on 24 host species. Neoseiulus tunus (De Leon 1967) occurred on 20 species. Stryphnodendron adstringens was the host plant that harbored the highest richness (eight species). No phytoseiid species were encountered on these plants: Anacardium occidentale, cf. Moraceae sp., Matayba elaeagnoides, Palicourea rigida, Piptocarpha rotundifolia, Rapanea sp., Tabebuia aurea and Tocoyena formosa.

\section{Subfamily AmblyseinnAe Muma, 1961}

\section{Amblyseius aerialis (Muma, 1955)}

Amblyseiopsis aerialis Muma 1955: 264; Garman 1958: 75.
Amblyseius aerialis; Athias-Henriot 1957: 338; Moraes et al. 1986: 6; 1991: 117; 2004: 13; Moraes and Mesa 1988: 71; Kreiter and Moraes 1997: 377; Feres and Moraes 1998: 126; Gondim Jr. and Moraes 2001: 67; Chant and McMurtry 2004: 203; 2007: 75; Feres et al. 2005: 45; Buosi et al. 2006: 3; Vasconcelos et al. 2006: 92; Guanilo et al. 2008a: 3; 2008b: 3; Mineiro et al. 2009: 40; Demite et al. 2011:34. Typhlodromus (Amblyseius) aerialis; Chant 1959: 88. Amblyseius (Amblyseius) aerialis; Denmark and Muma 1989: 15.

Origin of the material examined - Chapadão do Céu-GO (B): Myrtaceae sp. 1: I-10 (1); Myrtaceae sp. 3: X-09 (2).

\section{Amblyseius neochiapensis Lofego, Moraes and McMurtry, 2000}

Amblyseius neochiapensis Lofego et al. 2000: 462; Zacarias and Moraes 2001: 580; Chant and McMurtry 2004: 199; Lofego et al. 2004: 4; Moraes et al. 2004: 40; Demite et al. 2011:38.

Origin of the material examined - Chapadão do Céu-GO (B): Harconia speciosa: I-10: (1); Miconia albicans: XII-09 (1). Jataí-GO (C): Cf. Dilleniaceae sp.: VI-10 (3); Dimorphandra mollis: XII-09 (1); Genipa americana: I-10 (1), VI-10 (1); Miconia albicans: X-09 (1); Qualea grandiflora: X-09 (1), XII-09 (3), I-10 (2), VI-10 (6). Jataí-GO (D): Miconia albicans: XI-09 (1). Byrsonima intermedia: X-09 (3), XII-09 
(1); Davilla elliptica: X-09 (2); Miconia albicans: XII-09 (1); Sclerobium paniculatum: XII-09 (1); Xylopia aromatica: X-09 (2), XII-09 (4). Brasília-DF: Brosimum gaudichaudii: XII-09 (2), I-10 (1); cf. Dilleniaceae: XII09 (1), VI-10 (3); Echinolaena inflexa: VI-10 (3); Hymenaea stigonocarpa: VI-10 (8); Kielmeyera sp.: VI-10 (8); Plenckia populnea: VI-10 (6); Qualea grandiflora: I-10 (7); Roupala brasiliensis: XII-09 (2), VI-10 (5); Trachipogon sp.: I-10 (1), VI-10 (1). Unaí-MG (I): cf. Dilleniaceae sp.: I-10 (1); Chamaecrista orbiculata: X-09 (1), XII-09 (2); Duguetia furfuracea: X-09 (1); Ouratea spectabilis: X-09 (1), I-10 (1); Pouteria ramiflora: X-09 (1); Qualea grandiflora: XII-09 (8), I-10 (2); Stryphnodendron adstringens: XII-09 (1). Cristalina-GO (J): Annona crassiflora: I-10 (2), VI-10 (3); Davilla elliptica: VI-10 (6); Ouratea spectabilis: VI-10 (1); Qualea grandiflora: I-10 (1), VI-10 (5); Tabebuia ochracea: I-10 (1).

\section{Amblyseius sp.}

Origin of the material examined - Jataí-GO (C): Melinis minutiflora: XII-09 (1).

\section{cf. Graminaseius sp.}

Origin of the material examined - Brasília-DF (H): Echinolaena inflexa: I-10 (1). Cristalina-GO (J): Trachipogon sp.: XII-09 (1).

\section{Euseius citrifolius Denmark and Muma, 1970}

Euseius citrifolius Denmark and Muma 1970: 222; Moraes and McMurtry 1983: 138; Moraes et al. 1986: 38; 1991: 131; 2004: 64; Feres and Moraes 1998: 127; Gondim Jr. and Moraes 2001: 74; Zacarias and Moraes 2001: 580; Noronha and Moraes 2002: 1114; Lofego et al. 2004: 4; Demite et al. 2009: 47; 2011: 40.

Origin of the material examined - Chapadão do Sul-MS (A): Byrsonima intermedia: VI-10 (2); Caryocar brasiliense: XII-09 (1); Fuirena umbellata: VI-10 (1); Myrtaceae sp. 1: XII-09 (2). Chapadão do Céu-GO (B): Campomanesia pubescens: X-09 (5); Myrtaceae sp. 2: X-09 (4). Jataí-GO (C): Caryocar brasiliense: I-10 (1), VI-10 (3); Dimorphandra mollis: XII-09 (1); Genipa americana: VI-10 (2); Miconia albicans: XII09 (1); Stryphnodendron adstringens: X-09 (6), XII-09 (1), VI-10 (9); Xylopia aromatica: XII-09 (1), I-10 (1). Jataí-GO (D): Caryocar brasiliense: X-09 (1), XI-09 (1);
Harconia speciosa: XI-09 (4); Qualea grandiflora: X-09 (2), XI-09 (2). Rio Verde-GO (E): Sclerobium paniculatum: XII-09 (4). Edealina-GO (F): Erythroxylum deciduum: X-09 (2), XII-09 (5); Qualea grandiflora: XII09 (3). Brasília-DF (H): Caryocar brasiliense: X-09 (1), I-10 (1); Plenckia populnea: I-10 (1); Stryphnodendron adstringens: X-09 (12), I-10 (1), VI-10 (13).

\section{Euseius concordis (Chant, 1959)}

Typhlodromus (Amblyseius) concordis Chant 1959: 69. Amblyseius (Iphiseius) concordis; Muma 1961: 288. Amblyseius concordis; Chant and Baker 1965: 22. Euseius concordis; Denmark and Muma 1973: 264; Moraes and Oliveira 1982: 317; Moraes and McMurtry 1983: 138; Moraes et al. 1986: 39; 2004: 64; Feres and Moraes 1998: 127; Gondim Jr. and Moraes 2001: 74; Noronha and Moraes 2002: 1116; Lofego et al. 2004: 5; Demite et al. 2009: 48; 2011: 41. Euseius flechtmanni; Denmark and Muma 1970: 223; 1973: 261 (synonymy according to Moraes et al. 1982).

Origin of the material examined - Chapadão do Céu-GO (B): Harconia speciosa: VI-10 (2).

\section{Euseius plaudus Denmark and Muma, 1973}

Euseius plaudus Denmark and Muma 1973: 263. Moraes et al. 2004: 78.

Origin of the material examined - TupaciguaraMG (G): Caryocar brasiliense: XII-09 (1).

\section{Euseius sibelius (De Leon, 1962)}

Amblyseius (Typhlodromalus) sibelius De Leon 1962: 21. Euseius sibelius; Muma et al. 1970: 98; Moraes and McMurtry 1983: 140; Moraes et al. 1986: 54; 1999 [2000]: 243; 2004: 83; Moraes and Mesa 1988: 81; Feres and Moraes 1998: 128; Chant and McMurtry 2005a: 216; 2007: 123; Lofego et al. 2004: 6; 2009: 45; Guanilo et al. 2008a: 22; Feres et al. 2009: 467.Demite et al. 2011: 42. Euseius subalatus; De Leon 1965a: 127 (synonymy according to Muma et al. 1970).

Origin of the material examined - Jataí-GO (C): Dimorphandra mollis: X-09 (1); Qualea grandiflora: VI10 (2). Tupaciguara-MG (G): Sclerobium paniculatum: I-10 (2); Stryphnodendron adstringens: X-09 (8). Brasília-DF (H): Kielmeyera sp.: XII-09 (2), I-10 (1). Unaí-MG (I): cf. Dilleniaceae: XII-09 (1). 


\section{Iphiseiodes zuluagai Denmark and Muma, 1972}

Iphiseiodes zuluagai Denmark and Muma 1972: 23; 1973: 251; 1975: 287; Moraes et al. 1982: 18; 1986 : 61; 2004: 91; Aponte and McMurtry 1995: 165; Kreiter and Moraes 1997: 377; Feres and Moraes 1998: 127; Gondim Jr. and Moraes 2001: 76; Zacarias and Moraes 2001: 581; Lofego et al. 2004: 7; Guanilo et al. 2008a: 9; Demite et al. 2009: 48; 2011: 43.

Origin of the material examined - Chapadão do Céu-GO (B): Campomanesia pubescens: X-09 (2), XII09 (1), I-10 (2); Didymopanax vinosum: X-09 (2); Harconia speciosa: X-09 (3), VI-10 (2); Myrtaceae sp. 2: X09 (3), VI-10 (4); Myrtaceae sp. 3: VI-10 (1); Xylopia aromatica: VI-10 (6). Rio Verde-GO (E): Byrsonima coccolobifolia: XII-09 (2); Byrsonima intermedia: X-09 (1), XII-09 (2); Doliocarpus cf. dentatus: X-09 (2), XII09 (9); Ouratea spectabilis: X-09 (2). Cristalina-GO (J): Annona crassiflora: VI-10 (2); Kielmeyera cf. coriacea: VI-10 (1); Ouratea spectabilis: VI-10 (4); Qualea grandiflora: VI-10 (3).

\section{Neoparaphytoseius sooretamus (El-Banhawy, 1984)}

Amblyseius sooretamus El-Banhawy 1984: 128. Amblyseiulella sooretama; Moraes et al. 1986: 5. Neoparaphytoseius sooretamus; Chant and McMurtry 2003b: 215; Moraes et al. 2004: 98.

Origin of the material examined - Chapadão do Sul-MS (A): Byrsonima intermedia: XII-09 (1).

\section{Neoseiulus anonymus (Chant and Baker, 1965)}

Amblyseius anonymus Chant and Baker 1965: 21; Schicha and Elshafie 1980: 32; McMurtry 1983: 254. Neoseiulus anonymus; Denmark and Muma 1973: 27; Moraes and Mesa 1988: 76; Moraes et al., 1991: 126; Kreiter and Moraes 1997: 378; Moraes et al. 1999 [2000]: 245.

Origin of the material examined - Jataí-GO (C): Stryphnodendron adstringens: VI-10 (1). Brasília-DF (H): Stryphnodendron adstringens: VI-10 (1); Trachipogon sp.: X-09 (2), I-10 (2). Cristalina-GO (J): Trachipogon sp.: VI-10 (1).

\section{Neoseiulus benjamini (Schicha, 1981)}

Amblyseius benjamini Schicha 1981: 203; Schicha 1987: 119; Ueckermann and Loots 1988: 142. Neoseiulus benjamini; Beard 2001: 131; Chant and McMurtry 2003a: 27; Lofego et al. 2009: 46; Demite et al. 2011: 43.

Origin of the material examined - Jataí-GO (C): Trachipogon sp.: I-10 (3). Jataí-GO (D): Trachipogon sp.: XII-09 (3). Tupaciguara-MG (G): Stryphnodendron adstringens: XII-09 (1). Brasília-DF (H): cf. Dilleniaceae sp.: X-09 (4); Echinolaena inflexa: VI-10 (2); Trachipogon sp.: VI-10 (1).

\section{Neoseiulus idaeus Denmark and Muma, 1973}

Neoseiulus idaeus Denmark and Muma 1973: 266; Moraes et al. 1986: 83; 2004: 124; Chant and McMurtry 2003a: 21; 2007: 29.Amblyseius idaeus; Moraes and McMurtry 1983: 134; Demite et al. 2011: 43.

Origin of the material examined - Unaí-MG (I): Echinolaena inflexa: X-09 (2).

\section{Neoseiulus melinis Lofego and Moraes, 2003}

Neoseiulus melinis Lofego and Moraes 2003: 113; Moraes et al. 2004: 133; Lofego et al. 2009.

Origin of the material examined - Jataí-GO (C): Caryocar brasiliense: I-10 (1); Melinis minutiflora: I-10 (6); Trachipogon sp.: X-09 (4), XII-09 (2).

\section{Neoseiulus tunus (De Leon, 1967)}

Typhlodromips tunus De Leon 1967: 29; Denmark and Muma 1973: 253; Moraes et al. 1986: 151. Amblyseius tunus; McMurtry and Moraes 1989: 181; Feres and Moraes 1998: 126. Neoseiulus tunus; Ferla and Moraes 2002a: 872; 2002b: 1018; Chant and McMurtry 2003a: 21; 2007: 31; Moraes et al. 2004: 148; Lofego et al. 2004: 8; Bellini et al. 2005: 37; Feres et al. 2005: 45; Buosi et al. 2006: 5; Hernandes and Feres 2006: 4; Guanilo et al. 2008a: 29; 2008b: 21; Demite et al. 2009: 48; 2011: 44.

Origin of the material examined - Chapadão do Sul-MS (A): Bauhinia sp. 1: I-10 (4); Byrsonima pachyphylla: XII-09 (1), I-10 (2); Campomanesia pubescens: XII-09 (1); Didymopanax vinosum: XII-09 (1); Fuirena 
umbellata: I-10 (2); Miconia albicans: XII-09 (1); Pouteria torta: XII-09 (11). Chapadão do Céu-GO (B): Bauhinia sp. 2: XII-09 (1); Fuirena umbellata: XII09 (1). Jataí-GO (C): Caryocar brasiliense: I-10 (1). Rio Verde-GO (E): Doliocarpus cf. dentatus: XII-09 (2). Edealina-GO (F): Alibertia edulis: I-10 (3); Astronium sp.: XII-09 (6); Bauhinia sp. 3: XII-09 (2); Byrsonima verbascifolia: XII-09 (1); Davilla elliptica: XII-09 (2); Guettarda viburnoides: XII-09 (3); Qualea grandiflora: XII-09 (2), I-10 (2); Rhamnidium elaeocarpum: I-10 (1); Roupala brasiliensis: XII-09 (3), I-10 (1). Tupaciguara-MG (H): Miconia albicans: XII-09 (3); Qualea grandiflora: VI-10 (1); Roupala brasiliensis: VI-10 (8); Stryphnodendron adstringens: XII-09 (2).

\section{Proprioseiopsis dominigos (El-Banhawy, 1984)}

Amblyseius dominigos El-Banhawy 1984: 130; McMurtry and Moraes 1989: 185; Moraes et al. 1991: 126; Feres and Moraes 1998: 126. Proprioseiopsis dominigos; Moraes et al. 1986: 114; 2004: 175; Gondim Jr. and Moraes 2001: 81; Zacarias and Moraes 2001: 582; Chant and McMurtry 2005b: 15; 2007: 89; Buosi et al. 2006: 5; Hernandes and Feres 2006: 5; Guanilo et al. 2008a: 10; Demite et al. 2009: 48; 2011: 45; Mineiro et al. 2009: 42.

Origin of the material examined - Chapadão do Céu-GO (B): Xylopia aromatica: X-09 (4). Rio VerdeGO (E): Xylopia aromatica: XII-09 (1). Cristalina-GO (J): Trachipogon sp.: VI-10 (1).

\section{Proprioseiopsis ovatus (Garman, 1958)}

Amblyseiopsis ovatus Garman 1958: 78. Amblyseiulus ovatus; Muma 1961; Moraes and McMurtry 1983: 133; Moraes et al.1991: 127. Typhlodromus (Amblyseius) ovatus; Chant 1959: 90. Proprioseiopsis ovatus; Denmark and Muma 1973: 237; Moraes et al. 1986: 121; 2004: 184; Gondim Jr. and Moraes 2001: 82.

Origin of the material examined - Chapadão do Sul-MS (A): Byrsonima intermedia: XII-09 (2). Chapadão do Céu-GO (B): Myrtaceae sp. 2: X-09 (2); Qualea grandiflora: X-09 (1). Edealina-GO (F): Roupala brasiliensis: XII-09 (3).

\section{Ricoseius loxocheles (De Leon, 1965)}

Amblyseius (Ricoseius) loxocheles De Leon 1965a: 128. Ricoseius loxocheles; Denmark and Muma 1970:119; 1973: 249; Lofego et al. 2004: 9.

Origin of the material examined - Chapadão do Céu-GO (B): Myrtaceae sp. 1: XII-09 (2). CristalinaGO (J): Kielmeyera cf. coriacea: VI-10 (2).

\section{Typhlodromalus aripo De Leon, 1967}

Typhlodromalus aripo De Leon 1967: 21; Denmark and Muma 1973: 257; Moraes et al. 1986: 128; 1999 [2000]: 252; 2004: 195; Feres and Nunes 2001: 1255; Zacarias and Moraes 2001: 582; Chant and McMurtry 2005a: 199; 2007: 199; Feres et al. 2005: 46; Buosi et al. 2006: 6; Lofego et al. 2004: 10; 2009: 54; Demite et al. 2009: 49; 2011: 46. Amblyseius aripo; Moraes and McMurtry 1983: 132; Moraes and Mesa 1988: 73; Feres and Moraes 1998: 126.

Origin of the material examined - Rio VerdeGO (E): Byrsonima intermedia: XII-09 (2). CristalinaGO (J): Davilla elliptica: VI-10 (2).

\section{Typhlodromalus peregrinus (Muma, 1955)}

Typhlodromus peregrinus Muma 1955: 270. Typhlodromus (Amblyseius) peregrinus; Chant 1959: 97. Typhlodromalus peregrinus; Muma et al. 1970: 88; Moraes et al. 1986: 132; 2004: 202; Zacarias and Moraes 2001: 582; Chant and McMurtry 2005b: 199; 2007: 11. Amblyseius peregrinus; McMurtry 1983: 255; Moraes et al. 1991: 130. Typhlodromus (Amblyseius) robineae; Chant 1959: 98 (synonymy according to Muma 1964). Typhlodromus (Amblyseius) evansi; Chant 1959: 99 (synonymy according to Muma 1964). Typhlodromus (Amblyseius) primulae; Chant 1959: 99 (synonymy according to Muma 1964).

Origin of the material examined - Chapadão do Céu-GO (B): Myrtaceae sp. 2: X-09 (2).

Subfamily Phytoseinae Berlese, 1916

\section{Phytoseius guianensis De Leon, 1965}

Phytoseius guianensis De Leon 1965b: 18; Denmark and Muma 1973: 269; Moraes and McMurtry 1983: 144; Lofego et al. 2004: 11; Moraes et al. 2004: 239; 
Feres et al. 2005: 46; Chant and McMurtry 2007: 129; Guanilo et al. 2008b: 23. Phytoseius (Pennaseius) guianensis; Moraes et al. 1986: 211. Phytoseius (Phytoseius) guianensis; Denmark 1966: 23; Demite et al. 2011: 47.

Origin of the material examined - Rio VerdeGO (E): Xylopia aromatica: XII-09 (2).

\section{Phytoseius intermedius Evans and MacFarlane, 1962}

Phytoseius (Dubininellus) intermedius Evans and MacFarlane 1962: 588. Phytoseius (Phytoseius) intermedius; Ehara 1972: 170. Phytoseius intermedius; Moraes et al. 2004: 242; Chant and McMurtry 2007: 129; Ueckermann et al. 2007: 12; Demite et al. 2008: 17; 2011: 47. Phytoseius (Phytoseius) yira; Pritchard and Baker 1962: 227 (synonymy according to Denmark 1966).

Origin of the material examined - TupaciguaraMG (G): Miconia albicans: XII-09 (1). Brasília-DF (H): Caryocar brasiliense: VI-10 (1).

\section{Phytoseius nahuatlensis De Leon, 1959}

Phytoseius nahuatlensis De Leon 1959: 147; Chant and Baker 1965: 56; Feres and Moraes 1998: 128; Moraes et al. 2004: 248; Feres et al. 2005: 46; Chant and McMurtry 2007: 129; Demite et al. 2011: 48. Phytoseius (Phytoseius) nahuatlensis; Chant 1959: 106; Chant and Athias-Henriot 1960: 217; Denmark 1966: 25. Phytoseius (Pennaseius) nahuatlensis; De Leon 1965b: 14; Moraes et al. 1986: 213.

Origin of the material examined - Jataí-GO (C): Byrsonima pachyphylla: I-10 (1); Caryocar brasiliense: VI-10 (1); Xylopia aromatica: XII-09 (1), I-10 (1). Rio Verde-GO (E): Sclerobium paniculatum: XII-09 (8); Xylopia aromatica: X-09 (3), XII-09 (3). Edealina-GO (F): Astronium sp.: XII-09 (2); Byrsonima verbascifolia: X-09 (1); Caryocar brasiliense: X-09 (1), I-10 (1); Davilla elliptica: I-10 (1); Guettarda viburnoides: I-10 (1); Solanum lycocarpum: XII-09 (1). TupaciguaraMG (G): Caryocar brasiliense: I-10 (2); cf. Moraceae sp.: VI-10 (11); Sclerobium paniculatum: XII-09 (3), VI-10 (8); Stryphnodendron adstringens: XII-09 (1); Xylopia aromatica: X-09 (1), XII-09 (5), I-10 (1).

\section{SUBFAMILY TYPHLODROMINAE SCHEUTEN, 1857}

\section{Galendromus (Galendromus) annectens (De Leon, 1958)}

Typhlodromus annectens De Leon 1958: 78; Moraes and McMurtry 1983: 142; Moraes et al. 1991: 134. Galendromus annectens; Muma 1961: 298. Galendromus (Galendromus) annectens; Muma 1963: 30; Denmark and Muma 1973: 274; Moraes et al. 1982: 21; 2004: 265; Chant and McMurtry 2007: 167; Demite et al. 2011: 49.

Origin of the material examined - Jataí-GO (C): Stryphnodendron adstringens: I-10 (2). Rio Verde-GO (E): Himatanthus obovatus: XII-09 (2).

\section{Metaseiulus (Metaseiulus) ferlai Moraes, McMurtry and Lopes, 2006}

Metaseiulus (Metaseiulus) ferlai Moraes et al. 2006: 352; Chant and McMurtry 2007: 174; Demite et al. 2011: 50 .

Origin of the material examined - Rio VerdeGO (E): Caryocar brasiliense: X-09 (2). TupaciguaraMG (G): Tabebuia aurea: X-09 (1).

\section{Typhlodromus (Anthoseius) neobakeri Prasad, 1968}

Typhlodromus neobakeri Prasad 1968: 1369; Schicha 1987: 130. Amblydromella neobakeri; Tenorio et al. 1985: 303; Moraes et al. 1986: 168. Amblydromella (Aphanoseia) neobakeri; Denmark and Welbourn 2002: 308. Typhlodromus (Anthoseius) neobakeri; Moraes et al. 2004: 339; Lofego et al. 2009.

Origin of the material examined - Brasília-DF (H): Echinolaena inflexa: X-09 (1), I-10 (1), VI-10 (14); Trachipogon sp.: VI-10 (1).

\section{DISCUSSION}

Amblyseius neochiapensis and N. tunus were found on a large number of hosts (24 and 20 host species, respectively). However, Lofego and Moraes (2006) classified these mites as infrequent due to the small number of hosts on which they were found, in the periphery of the Cerrado, in São Paulo. Demite et al. (2009) also found N. tunus in peripheral areas of 
Cerrado at Mato Grosso, but associated with only one host. The differences between the results of this study and the above cited may be related to geographical position, climatic differences or floristic composition among fragments, which may alter species composition from one region to another (Felfili et al. 2002; Reis et al. 2000; Bridgewater et al. 2004).

According to some authors, leaf structures are important factors determining the composition of phytoseiid species in plants (Beard and Walter 2001; Hadamar et al. 1986; Moraes et al. 1993). Our data partially agree with this statement, since $27 \%$ of mite species did not occur in more than one host, suggesting a very specific mite-host relationship. Furthermore, some species groups had preference for some kind of leaf structure. For example, species of the genus Phytoseius almost always occurred in plants with trichomes, such as $C$. brasiliense and $X$. aromatica.

However, other species did not show any preference at all for specific host. Amblyseius neochiapensis, E. citrifolius, and N. tunus occurred in plants with diverse leaf structures, such as smooth leaves of M. albicans, leaves with trichomes of Q. grandiflora and leaves with domatia of S. adstringens. Lofego and Moraes (2006) found similar results in CerradoAtlantic forest transition area, in which Amblyseius acalyphus Denmark and Muma, 1973 occurred in host with different foliar structures, whereas the majority of Transeius belotti (Moraes and Mesa), 1988 occurred in only one host.

Therefore, we conclude that the occurrence of some phytoseiid in the Cerrado may be restricted to some plant species. This restricted distribution is likely related to foliar structures or feeding resources available in the hosts. Nonetheless, other species apparentely are not affected by these characteristics, and occur in a wide variety of plants in the region. Taken together, these results point to the highly complex distribution of phytoseiid mites in the Cerrado and reinforce the importance of the conservation of this biome. Most of species found in this study (17 in total) were previously recorded in Atlantic Forest remnants (Castro and Moraes 2010; Demite et al. 2011; Feres and Moraes 1998; Feres et al. 2005; Zacarias and Moraes 2002). Comparing our results with previous records from peripheral areas of Cerrado (Lofego et al. 2004; Lofego and Moraes 2006; Demite et al. 2009), only 12 species are common between these two areas.

In conclusion, the results suggest that few Phytoseiidae species are endemic from Cerrado, a fact also pointed out by Tixier and Kreiter (2009). However, due to the small number of surveys and the high rate of endemic plants in this biome (Myers et al. 2000), these results show probably only a fraction of the real diversity of phytoseiid in the region, since the richness of animal groups tend to increase as plant diversity increases (Brose 2003; Dennis et al. 1998; MacArthur and MacArthur 1961). Therefore, more surveys should be conducted in the Cerrado, including physiognomies and hosts not considered in this study. This would improve the knowledge about phytoseiid species richness from this Neotropical savannah.

\section{ACKNOWLEDGEMENTS}

We would like to thank FAPESP (Fundação de Amparo à Pesquisa do Estado de São Paulo) (Procs. 06/57868-9 and 08/07835-2) and CAPES (Coordenação de Aperfeiçoamento de Pessoal de Nível Superior) for the financial support to this project. We also would like to thank Dr. Andréia A. Rezende and Prof. Dr. Neusa T. Ranga (UNESP, São José do Rio Preto) for native plant identifications, and finally to land owners for providing access to the forest fragments in their properties.

\section{REFERENCES}

Aponte O., McMurtry J.A. 1995 - Revision of the genus Iphiseiodes De Leon (Acari: Phytoseiidae) - Int. J. Acarol., 21: 165-183. doi:10.1080/01647959508684056

Athias-Henriot C. 1957 - Phytoseiidae et Aceosejidae (Acarina, Gamasina) d'Algerie. I. Genres Blattisocius Keegan, Iphiseius Berlese, Amblyseius Berlese, Phytoseius Ribaga, Phytoseiulus Evans - Bull. Soc. Hist. Nat. Afr. N., 48: 319-352.

Beard J.J. 2001 - A review of Australian Neoseiulus Hughes and Typhlodromips De Leon (Acari: Phytoseiidae: Amblyseiinae) — Invert. Taxon., 15: 73-158. doi:10.1071/IT99017 
Beard J.J., Walter G.H. 2001 - Host plant specificity in several species of generalist mite predators Ecol. Entomol., 26: 562-570. doi:10.1046/j.13652311.2001.00367.x

Bellini M.R., Moraes G.J. de, Feres R.J.F. 2005 — Plantas de ocorrência espontânea como substrato alternativos para fitoseídeos (Acari, Phytoseiidae) em cultivos de seringueira Hevea brasiliensis Muell. Arg. (Euphorbiaceae) - Rev. Bras. Zool., 22: 35-42. doi:10.1590/S0101-81752005000100005

Bridgewater S., Ratter J.A., Ribeiro J.F. 2004 - Biogeographic patterns, $\beta$-diversity and dominance in the Cerrado biome of Brazil - Biodivers. Conserv., 13: 2295-2318. doi:10.1023/B:BIOC.0000047903.37608.4c

Brose U. 2003 - Bottom-up control of carabid beetle communities in early successional wetlands: mediated by vegetation structure or plant diversity? - Oecologia, 135: 407-413.

Buosi R., Feres R.J.F., Oliveira A.R., Lofego A.C., Hernandes F.A. 2006 - Ácaros plantícolas (Acari) da "Estação Ecológica de Paulo de Faria", Estado de São Paulo, Brasil - Biota Neotrop., 6: 1-20.

Castro T.M.M.G. de, Moraes G.J. de 2010 — Diversity of phytoseiid mites (Acari: Mesostigmata: Phytoseiidae) in the Atlantic Forest of São Paulo - Syst. Biodivers., 8: 301-307. doi:10.1080/14772001003801375

Chant D.A. 1959 - Phytoseiid mites (Acarina: Phytoseiidae). Part I. Bionomics of seven species in southeastern England. Part II. A taxonomic review of family Phytoseiidae, with descriptions of 38 new species - Can. Entomol., Suppl., 91: 1-166. doi:10.4039/entm9112fv

Chant D.A., Athias-Henriot C. 1960 - The genus Phytoseius Ribaga, 1902 (Acarina: Phytoseiidae) - Entomophaga, 5: 213-228. doi:10.1007/BF02383272

Chant D.A., Baker E.W. 1965 - The Phytoseiidae (Acarina) of Central America - Mem. Entomol. Soc. Can., 41: 1-56. doi:10.4039/entm9741fv

Chant D.A., McMurtry J.A. 2003a - A review of the subfamily Amblyseiinae Muma (Acari: Phytoseiidae): Part I. Neoseiulini new tribe - Int. J. Acarol., 29: 3-46. doi:10.1080/01647950308684319

Chant D.A., McMurtry J.A. 2003b - A review of the subfamily Amblyseiinae Muma (Acari: Phytoseiidae): Part II. The tribe Kampimodromini Kolodochka - Int. J. Acarol., 29: 179-224. doi:10.1080/01647950308684331

Chant D.A., McMurtry J.A. 2004 - A review of the subfamily Amblyseiinae Muma (Acari: Phytoseiidae): Part III. The tribe Amblyseiini Wainstein, subtribe Amblyseiina n. subtribe - Int. J. Acarol., 30: 171-228. doi:10.1080/01647950408684388
Chant D.A., McMurtry J.A. 2005a - A review of the subfamily Amblyseiinae Muma (Acari: Phytoseiidae): Part VI. The tribe Euseiini n. tribe, subtribes Typhlodromalina n. subtribe, Euseiina n. subtribe, and Ricoseiina n. subtribe - Int. J. Acarol., 31: 187-224. doi:10.1080/01647950508684424

Chant D.A., McMurtry J.A. 2005b - A review of the subfamily Amblyseiinae Muma (Acari: Phytoseiidae): Part V. Tribe Amblyseiini, subtribe Proprioseiopsina Chant and McMurtry - Int. J. Acarol., 31: 3-22. doi:10.1080/01647950508684412

Chant D.A., McMurtry J.A. 2007 - Illustrated keys and diagnoses for the genera and subgenera of the Phytoseiidae of the world (Acari: Mesostigmata) - Michigan: Indira Publishing House. pp. 219.

Daud R.D., Feres R.J.F., Bousi R. 2007 — Ácaros (Arachnida: Acari) Associados a Bauhinia variegata L. (Leguminosae) no Noroeste do Estado de São Paulo Neotrop. Entomol., 36: 322-325. doi:10.1590/S1519566X2007000200025

De Leon D. 1958 - Four new Typhlodromus from southern Florida (Acarina: Phytoseiidae) - Fla. Entomol., 41: 73-76. doi:10.2307/3492363

De Leon D. 1959 - A new genus and three new species of phytoseiid mites from Mexico with collection records on Phytoseius plumifer (C. and F.) and P. macropilis (Banks) - Entomol. News, 70: 147-152.

De Leon D. 1962 - Twenty-three new phytoseiids, mostly from southeastern United States - Fla. Entomol., 45: 11-27. doi:10.2307/3492899

De Leon D. 1965a - Phytoseiid mites from Puerto Rico with descriptions of new species (Acarina: Mesostigmata) - Fla. Entomol., 48: 121-131. doi:10.2307/3493102

De Leon D. 1965b - Ten new species of Phytoseius (Pennaseius) from Mexico, Trinidad, and British Guiana with a key to species (Acarina: Phytoseiidae) - Entomol. News, 76: 11-21.

De Leon D. 1967 - Some mites of the Caribbean Area. Part I. Acarina on plants in Trinidad, West Indies. Lawrence: Allen Press Inc. pp. 66.

Demite P.R., Lofego A.C., Feres R.J.F. 2008 - Three new species of Phytoseius Ribaga (Acari: Phytoseiidae), and a new record from Brazil — Zootaxa, 1909: 16-26.

Demite P.R., Feres R.J.F., Lofego A.C., Oliveira A.R. 2009 - Plant inhabiting mites (Acari) from the Cerrado biome of Mato Grosso State, Brazil - Zootaxa, 2061: 45-60.

Demite P.R., Lofego A.C., Feres R.J.F. 2011 — Phytoseiidae (Acari) in Forest fragments in the State of São Paulo, Brazil - Zootaxa, 3089: 31-56. 
Denmark H.A. 1966 - Revision of the genus Phytoseius Ribaga, 1904 (Acarina: Phytoseiidae) - Fla. Dep. Agric. Bull., 6: 1-105.

Denmark H.A., Muma M.H. 1970 - Some phytoseiid mites of Paraguay (Phytoseiidae: Acarina) - Fla. Entomol., 53: 219-227. doi:10.2307/3493192

Denmark H.A., Muma M.H. 1972 - Some Phytoseiidae of Colombia (Acarina: Phytoseiidae) - Fla. Entomol., 55: 19-29. doi:10.2307/3493637

Denmark H.A., Muma M.H. 1973 - Phytoseiidae mites of Brazil (Acarina, Phytoseiidae) - Rev. Bras. Biol., 33: 235-276.

Denmark H.A., Muma M.H. 1975 - The Phytoseiidae (Acarina: Mesostigmata) of Puerto Rico - J. Agr. Univ. Puert. Rico, 59: 279-304.

Denmark H.A., Muma M.H. 1989 - A revision of the genus Amblyseius Berlese, 1914 (Acari: Phytoseiidae) - Occas. Pap. Fla. State Collect. Arthropods, 4: 1149.

Denmark H.A., Welbourn W.C. 2002 - Revision of the genera Amblydromella Muma and Anthoseius De Leon (Acari: Phytoseiidae) - Int. J. Acarol., 28: 291-316. doi:10.1080/01647950208684308

Dennis P., Young M.R., Gordon I.J. 1998 — Distribution and abundance of small insects and arachnids in relation to structural heterogeneity of grazed, indigenous grasslands - Ecol. Entomol., 23: 253-264. doi:10.1046/j.1365-2311.1998.00135.x

Ehara S. 1972 - Some phytoseiid mites from Japan, with descriptions of thirteen new species (Acarina: Mesostigmata) - Mushi, 46: 137-173.

El-Banhawy E.M. 1984 — Description of some phytoseiid mites from Brazil (Acarina: Phytoseiidae) - Acarologia, 25: 125-144.

Evans G.O., MacFarlane D. 1962 - A new mites of the genus Phytoseius Ribaga (Acari: Mesostigmata) Annu. Mag. Nat. Hist., Ser. 13, 4: 587-588.

Farias A.R.N., Flechtmann C.H.W., Moraes G.J.de, McMurtry J.A. 1981 - Predadores do ácaro verde da mandioca, no nordeste do Brasil - Pesq. Agropec. Bras., 16: 313-317.

Felfili J.M., Nogueira P.E., Silva Júnior M.C. da, Marimon B.S., Delitti W.B.C. 2002 - Composição florística e fitossociologia do cerrado sentido restrito no município de Água Boa-MT — Acta Bot. Bras., 16: 103-112.

Feres R.J.F., Moraes G.J. 1998 — Phytoseiid mites (Acari: Phytoseiidae) from woody areas in the state of São Paulo, Brazil - Syst. Appl. Acarol., 3: 125-132.

Feres R.J.F., Nunes M.A. 2001 - Ácaros (Acari, Arachnida) associados à euforbiáceas nativas em áreas de cultivo de seringueiras (Hevea brasiliensis Muell. Arg.,
Euphorbiaceae) na região noroeste do Estado de São Paulo, Brasil - Rev. Bras. Zool., 18: 1253-1264. doi:10.1590/S0101-81752001000400020

Feres R.J.F., Lofego A.C., Oliveira A.R. 2005 - Ácaros plantícolas (Acari) da "Estação Ecológica do Noroeste Paulista", Estado de São Paulo, Brasil — Biota Neotrop., 5: 1-14.

Feres R.J.F., Vieira M.R., Daud R.D., Pereira Jr. E.G., Oliveira G.F., Dourado C. 2009 - Ácaros (Arachnida, Acari) de plantas ornamentais na região noroeste do Estado de São Paulo, Brasil: inventário e descrição dos sintomas causados pelos fitófagos - Rev. Bras. Entomol., 53: 466-474. doi:10.1590/S008556262009000300024

Ferla N.J., Moraes G.J. 2002a — Ácaros (Arachnida, Acari) da seringueira (Hevea brasiliensis Muell. Arg.) no Estado de Mato Grosso, Brasil - Rev. Bras. Zool., 19: 867-888. doi:10.1590/S0101-81752002000300025

Ferla N.J., Moraes G. J. 2002b - Ácaros predadores (Acari) em plantas nativas e cultivadas do Estado do Rio Grande do Sul, Brasil — Rev. Bras. Zool., 19: 10111031. doi:10.1590/S0101-81752002000400006

Fiaboe K.K.M., Gondim M.G.C. Jr., Moraes G.J. de, Ogol C.K.P.O., Knapp. M. 2007 - Surveys for natural enemies of the tomato red spider mite Tetranychus evansi (Acari: Tetranychidae) in the northeastern and southeastern Brazil - Zootaxa, 1395: 33-58.

Furtado I.P., Kreiter S., Moraes G.J. de, Tixier M.-S., Flechtmann C.W.H., Knapp M. 2004 [2005] — Plant mites from northeastern Brazil, with descriptions of two new species of the family Phytoseiidae (Mesostigmata) - Acarologia, 45: 131-143.

Furtado I.P., Moraes G.J. de, Kreiter S., Knapp M. 2006 - Search for effective natural enemies of Tetranychus evansi in south and southeast Brazil - Exp. Appl. Acarol., 40: 157-174. doi:10.1007/s10493-006-9045-y

Furtado I.P., Toledo S. E., Moraes G.J. de, Kreiter S., Knapp M. 2007 - Search for effective natural enemies of Tetranychus evansi (Acari: Tetranychidae) in northwest Argentina - Exp. Appl. Acarol., 43: 121-127. doi:10.1007/s10493-007-9104-z

Garman P. 1958 - New species belonging to the genera Amblyseius and Amblyseiopsis with keys to Amblyseius, Amblyseiopsis and Phytoseiulus - Ann. Entomol. Soc. Am., 51: 69-79.

Gerson U., Smiley R.L., Ochoa R. - Mites (Acari) for pest control — Oxford: Blackwell Science Ltd. pp. 539.

Gondim Jr. M.G.C., Moraes G.J. 2001 — Phytoseiid mites (Acari: Phytoseiidae) associated with palm trees (Arecaceae) in Brazil — Syst. Appl. Acarol., 6: 65-94.

Guanilo A.D., Moraes G.J. de, Knapp M. 2008a — Phytoseiid mites (Acari: Phytoseiidae) of the subfamily Am- 
blyseiinae Muma from Peru, with description of four new species - Zootaxa, 1880: 1-47.

Guanilo A.D., Moraes G.J. de, Knapp M. 2008b - Phytoseiid mites (Acari: Phytoseiidae) from Argentina, with description of a new species - Zootaxa, 1884: 1-35.

Guanilo A.D., Moraes G.J. de, Toledo S., Knapp, M. 2008c - Phytoseiid mites (Acari: Phytoseiidae) from Argentina, with description of a new species - Zootaxa, 1884: 1-35.

Guanilo A.D., Moraes, G.J. de, Toledo S., Knapp M. 2010 - New records of Tetranychus evansi and associated natural enemies in northern Argentina - Syst. Appl. Acarol., 15: 3-20.

Hadamar J.J., AliNiazee M.T., Croft B.A. 1986 - Phytoseiid mites of major crops in the Willamette Valey, OR, and pesticide resistance in Typhlodromus pyri Scheuten — Environ. Entomol., 15: 1255-1262.

Hernandes F.A. and Feres R.J.F. 2006 - Review about mites (Acari) of rubber trees (Hevea spp., Euphorbiaceae) in Brazil — Biota Neotrop., 6: 1-24.

Kreiter S., Moraes G.J. 1997 - Phytoseiid mites (Acari: Phytoseiidae) from Guadeloupe and Martinique Fla. Entomol., 80: 376-382. doi:10.2307/3495770

Lawson-Balagbo L.M., Gondim Jr. M.G.C., Moraes G.J. de, Hanna R., Schausberger P. 2008 - Exploration of the acarine fauna on coconut palm in Brazil with emphasis on Aceria guerreronis (Acari: Eriophyidae) and its natural enemies - Bull. Entomol. Res., 98: 83-96. doi:10.1017/S0007485307005421

Lofego A.C., Moraes G.J. 2003 - Two new species of Neoseiulus Hughes (Acari: Phytoseiidae) from Brazil - Int. J. Acarol., 29: 113-117. doi:10.1080/01647950308683647

Lofego A.C., Moraes, G.J. 2006 - Ácaros (Acari) associados à Mirtáceas (Myrtaceae) em áreas de Cerrado no Estado de São Paulo com análise faunística das famílias Phytoseiidae e Tarsonemidae Neotrop. Entomol., 35: 731-746. doi:10.1590/S1519566X2006000600003

Lofego A.C., Moraes G.J. de, Castro L.A.S. 2004 - Phytoseiid mites (Acari: Phytoseiidae) on Myrtaceae in the State of São Paulo, Brazil - Zootaxa, 516: 1-18.

Lofego A.C., Moraes G.J. de, McMurtry J.A. 2000 - Three new species of phytoseiid mites (Acari: Phytoseiidae) from Brazil - An. Soc. Entomol. Bras., 29: 461-467. doi:10.1590/S0301-80592000000300008

Lofego A.C., Demite P.R., Kishimoto R.G., Moraes, G.J. 2009 - Phytoseiid mites on grasses in Brazil (Acari: Phytoseiidae) - Zootaxa, 2240: 41-59.

MacArthur R. H., MacArthur J. W. 1961 — On bird species diversity - Ecology, 42: 594-598.
McMurtry J.A. 1983 - Phytoseiidae mites from Guatemala, with descriptions of two new species and redefinitions of the genera Euseius, Typhloseiopsis, and Typhlodromus occidentalis species group (Acari: Mesostigmata) — Int. J. Entomol., 25: 249-272.

McMurtry J.A. and Croft B.A. 1997 - Life-styles of phytoseiid mites and their roles in biological control - Annu. Rev. Entomol., 42: 291-321. doi:10.1146/annurev.ento.42.1.291

McMurtry J.A., Moraes G.J. de 1989 — Some phytoseiid mites from Peru with descriptions of four new species (Acari: Phytoseiidae) - Int. J. Acarol., 15: 179-188. doi:10.1080/01647958908683843

Mineiro J.L. de C., Raga A., Sato M.E., Lofego A.C. 2009 Ácaros associados ao cafeeiro (Coffea spp.) no estado de São Paulo, Brasil. Parte I. Mesostigmata - Biota Neotrop., 9: 37-46.

Ministério do Meio Ambiente 2007 - Cerrado e Pantanal: áreas e ações prioritárias para conservação da Biodiversidade - Brasília: Ministério do Meio Ambiente. pp. 397.

Moraes G.J. de, Flechtmann C.H.W. 2008 - Manual de Acarologia: acarologia básica e ácaros de plantas cultivadas no Brasil - Ribeirão Preto: Holos Editora. pp. 228.

Moraes G.J. de, McMurtry J. A. 1983 - Phytoseiid mites (Acarina) of northeastern Brazil with descriptions of four new species — Int. J. Acarol., 9: 131-148. doi:10.1080/01647958308683326

Moraes G.J. de, Mesa N.C. 1988 - Mites of the family Phytoseiidae (Acari) in Colombia, with descriptions of three new species - Int. J. Acarol., 14: 71-88. doi:10.1080/01647958808683790

Moraes G.J. de, Oliveira J.V. 1982 - Phytoseiid mites of coastal Pernambuco in northeastern Brazil - Acarologia, 23: 315-318.

Moraes G.J. de, Denmark H.A., Guerrero J.M. 1982 - Phytoseiidae mites of Colombia (Acarina: Phytoseiidae) - Int. J. Acarol., 8: 15-22. doi:10.1080/01647958208683273

Moraes G.J de, Kreiter S., Lofego A.C. 1999 [2000] — Plant mites (Acari) of the French Antilles. 3. Phytoseiidae (Gamasida) - Acarologia, 40: 237-264.

Moraes G.J. de, McMurtry J.A., Denmark, H.A. 1986 A catalog of the mite family Phytoseiidae: references to taxonomy, synonymy, distribution and habitat Brazil: Embrapa-DDT. pp. 356.

Moraes G.J. de, McMurtry J.A., Lopes P.C. 2006 - Redefinition of Metaseiulus Muma (Acari: Phytoseiidae) and description of a new species from Brazil - Int. J. Acarol., 32: 351-354. doi:10.1080/01647950608684481 
Moraes G.J. de, Mesa N.C., Braun A. 1991 - Some phytoseiid mites of Latin America (Acari: Phytoseiidae) - Int. J. Acarol., 17: 117-139. doi:10.1080/01647959108683892

Moraes G.J. de, de Alencar J.A., Lima J.L.S., Yaninek J.S. 1993 - Alternative plant habitats for common phytoseiid predators of the cassava green mite (Acari: Phytoseiidae. Tetranychidae) in northeast Brazil - Exp. Appl. Acarol., 17: 77-90.

Moraes G.J. de, McMurtry J.A., Denmark H.A., Campos C.B. $2004-$ A revised catalog of the mite family Phytoseiidae - Zootaxa, 434: 1-494.

Muma M.H. 1955 - Phytoseiidae (Acarina) associated with citrus in Florida - Ann. Entomol. Soc. Am., 48: 262-272.

Muma M.H. 1961 - Subfamilies, genera and species of Phytoseiidae (Acarina: Mesostigmata) - Bull. Fla. State Museum, Biol. Science, 5: 267-302.

Muma M.H. 1963 - The genus Galendromus Muma, 1961 (Acarina: Phytoseiidae) - Fla. Entomol., Suppl. 1: 15-41. doi:10.2307/3493355

Muma M.H. 1964 - Annotated list and keys to Phytoseiidae (Acarina: Mesostigmata) associated with Florida citrus - Fl. Agric. Exp. Stn. Bull., 685: 1-42.

Muma M.H., Denmark H.A., De Leon D. 1970 - Phytoseiidae of the Florida. Arthropods of Florida and neighboring land areas — USA: Florida Department of Agriculture and Consumer Services, Division of Plant Industry. pp. 150.

Myers N., Mittermeier R.A., Mittermeier C.G., da Fonseca G.A.B., Kent J. 2000 - Biodiversity hotspots for conservation priorities - Nature, 403: 853-858.

Noronha A.C. da S., Moraes G.J. 2002 — Variações morfológicas intra e interpopulacionais de Euseius citrifolius Denmark and Muma e Euseius concordis (Chant) (Acari, Phytoseiidae) - Rev. Bras. Zool., 19: 11111122.

Prasad V. 1968 - Some Typhlodromus mites from Hawaii — Ann. Entomol. Soc. Am., 61: 1369-1372.

Pritchard A.E., Baker E.W. 1962 - Mites of the family Phytoseiidae from Central Africa, with remarks on genera of the world - Hilgardia, 33: 205-309.

Reis P.R., Chiavegato L.G., Alves E.B., Sousa, E.O. 2000 Ácaros da Família Phytoseiidae Associados aos Citros no Município de Lavras, Sul de Minas Gerais - An. Soc. Entomol. Bras., 29: 95-104. doi:10.1590/S030180592000000100012

Ribeiro J.F., Walter B.M.T. 1998 - Fitofisionomias do bioma Cerrado - In: Sano M.S., Almeida, S.P. (Eds), Cerrado: ambiente e flora. Brasília: Embrapa. p. 89106.

Rosa A.A., Gondim M.G.C.Jr., Fiaboe K.K.M., Moraes G.J. de, Knapp M. 2005 — Predatory mites associated with Tetranychus evansi Baker and Pritchard (Acari: Tetranychidae) on native solanaceous plants of coastal Pernambuco State, Brazil - Neotrop. Entomol., 34: 689-692.

Schicha E. 1981 - A new species of Amblyseius (Acarina: Phytoseiidae) from Australia compared with ten closely related species from Asia, America and Africa - Int. J. Acarol., 7: 203-216. doi:10.1080/01647958108683262

Schicha E. 1987 - Phytoseiidae of Australia and neighboring areas - Michigan: Indira Publishing House. pp. 187.

Schicha E., Elshafie M. 1980 - Four new species of phytoseiid mites from Australia, and three species from America redescribed (Acari: Phytoseiidae) - J. Aust. Entomol. Soc., 19: 27-36. doi:10.1111/j.14406055.1980.tb00957.x

Tenorio J.M., Denmark H.A., Swift S.F. 1985 - Catalog of Acari in the Hawaiian Islands. I. Mesostigmata (or Gamasida) (Acari) — Int. J. Entomol., 27: 297-309.

Tixier M.-S., Kreiter S. 2009 - Arthropods in biodiversity hotspots: the case of Phytoseiidae (Acari: Mesostigmata) - Biodivers. Conserv., 18: 507-527. doi:10.1007/s10531-008-9517-y

Ueckermann E.A., Loots G.C. 1988 - The African species of the subgenera Anthoseius De Leon and Amblyseius Berlese (Acari: Phytoseiidae) — Entomol. Mem., Depart. Agric. Water Supply, Repub. S. Afr., 73: 1-168.

Ueckermann E.A., Zannou I.D., Moraes G.J. de, Oliveira A.R., Hanna R., Yaninek J.S. 2007 — Phytoseiid mites of the subfamily Phytoseiinae (Acari: Phytoseiidae) from sub-Saharan Africa - Zootaxa, 1658: 1-20.

Vasconcelos D.E., Silva F.R., Barbosa D.G.F., Gondim Jr. M.G.C., Moraes G.J. 2006 - Diversidade de fitoseídeos (Acari: Phytoseiidae) em fruteiras tropicais no Estado de Pernambuco, Brasil — Magistra, 18: 90101.

Zacarias M.S., Moraes G.J. 2001 - Phytoseiid mites (Acari) associated with rubber trees and other euphorbiaceous plants in southeaster Brazil Neotrop. Entomol., 30: 579-586. doi:10.1590/S1519566X2001000400011

Zacarias M.S., Moraes G.J. 2002 — Mite diversity (Arthropoda: Acari) on euphorbiaceous plants in three localities in the State of São Paulo — Biota Neotrop., 2: 1-12.

\section{COPYRIGHT}

$(c)$ EY-No-ND Rezende and Lofego. Acarologia is under free license. This open-access article is distributed under the terms of the Creative Commons-BY-NC-ND which permits unrestricted non-commercial use, distribution, and reproduction in any medium, provided the original author and source are credited. 